\title{
GCU
}

Glasgow Caledonian

University

University for the Common Good

\section{Matched population-based study examining the risk of type 2 diabetes in people with and without diagnosed hepatitis $C$ virus infection}

Schnier, Christian; Wild, S.; Kurdi, Z.; Povey, C.; Goldberg, David J.; Hutchinson, Sharon J.

Published in:

Journal of Viral Hepatitis

DOI:

$10.1111 /$ jvh. 12520

Publication date:

2016

Document Version

Author accepted manuscript

Link to publication in ResearchOnline

Citation for published version (Harvard):

Schnier, C, Wild, S, Kurdi, Z, Povey, C, Goldberg, DJ \& Hutchinson, SJ 2016, 'Matched population-based study examining the risk of type 2 diabetes in people with and without diagnosed hepatitis $C$ virus infection', Journal of Viral Hepatitis, vol. 23, no. 8, pp. 596-605. https://doi.org/10.1111/jvh.12520

\section{General rights}

Copyright and moral rights for the publications made accessible in the public portal are retained by the authors and/or other copyright owners and it is a condition of accessing publications that users recognise and abide by the legal requirements associated with these rights.

Take down policy

If you believe that this document breaches copyright please view our takedown policy at https://edshare.gcu.ac.uk/id/eprint/5179 for details

of how to contact us. 
1 Title:

2 Matched population-based study examining the risk of type 2 diabetes in

3 people with and without diagnosed hepatitis $\mathrm{C}$ infection

5 Running title:

6 Type 2 diabetes and HCV

8 Authors:

9 C. Schnier, School of Health and Life Sciences, Glasgow Caledonian

10 University, Glasgow G4 0BA \& Health Protection Scotland, Meridian

11 Court, 5 Cadogan Street, Glasgow G2 6QE, UK

12 S. Wild, Centre for Population Health Sciences, University of

13 Edinburgh, Edinburgh EH8 9AG

14 Z. Kurdi, Centre for Population Health Sciences, University of

15 Edinburgh, Edinburgh EH8 9AG

16 C. Povey, Information Service Division, Scotland, 1 South Gyle

17 Crescent, Edinburgh EH12 9EB, UK

18 D. J. Goldberg, Health Protection Scotland, Meridian Court, 5 Cadogan

19 Street, Glasgow G2 6QE, UK

20 S. J. Hutchinson, School of Health and Life Sciences, Glasgow

21 Caledonian University, Glasgow G4 0BA \& Health Protection Scotland,

22 Meridian Court, 5 Cadogan Street, Glasgow G2 6QE, UK

24 Corresponding Author:

25 Sharon Hutchinson

26 School of Health and Life Sciences

27 Glasgow Caledonian University

28 Cowcaddens Road

29 Glasgow

30 G4 0BA

31 sharon.hutchinson@gcu.ac.uk

32 Tel: +44(0)141 2731949 


\section{Matched population-based study examining}

36 the risk of type 2 diabetes in people with and

37 without diagnosed hepatitis $\mathrm{C}$ infection

\section{Abstract}

39 Meta-analyses have found hepatitis C virus (HCV) infection to be

40 associated with an increased risk of type 2 diabetes mellitus (T2DM).

41 Here, we examine this association within a large population-based study,

42 according to RNA status.

43 A data-linkage approach was used to examine the excess risk of 44 diagnosed T2DM in people diagnosed with HCV-antibodies in Scotland

45 (21,929 $\mathrm{Ab}^{+v e s}$; involving 15,827 RNA ${ }^{+v e s}, 3927 \mathrm{RNA}^{-v e s}$ and 2175

46 with unknown RNA-status) compared to that of a three-fold larger

47 general population sample matched for sex, age and postcode $(65,074$

$48 \mathrm{Ab}^{-v e s}$ ). To investigate effects of ascertainment bias the following

49 periods were studied: up to one year before (pre-HCV)/within one year

50 of $($ peri-HCV)/more than one year post (post-HCV) the date of

51 HCV-diagnosis. 
52 T2DM had been diagnosed in $2.9 \%$ of $\mathrm{Ab}^{+v e s}$ (including $3.2 \%$ of

$53 \mathrm{RNA}^{+\mathrm{ves}}$ and $2.3 \%$ of $\mathrm{RNA}^{-\mathrm{ves}}$ ) and $2.7 \%$ of $\mathrm{Ab}^{-v e s}$. A higher proportion

54 of T2DM was diagnosed in the peri-HCV period (i.e. around the time of

55 HCV-diagnosis) for the $\mathrm{Ab}^{+v e s}$ (22\%) compared to $\mathrm{Ab}^{-v e s}$ (10\%). In

56 both the pre-HCV and post-HCV periods, only those $\mathrm{Ab}^{+v e s}$ living in

57 less deprived areas (13\% of the cohort) were found to have a significant

58 excess risk of T2DM compared to $\mathrm{Ab}^{- \text {ves }}$ (adjusted odds ratio in the

59 pre-HCV period: 4.0 for females and 2.3 for males; adjusted hazard ratio

60 in the post-HCV period: 1.5). These findings were similarly observed for

61 both $\mathrm{RNA}^{+v e s}$ (chronic) and $\mathrm{RNA}^{-v e s}$ (resolved).

62 In the largest study of T2DM among chronic HCV-infected individuals

63 to date, there was no evidence to indicate that infection conveyed an

64 appreciable excess risk of T2DM at the population level.

66 Keywords:

67 Hepatitis C, Type 2 Diabetes, Matched cohort study, Data linkage

681 Introduction

69 A large consistent body of evidence from several observational studies

70 suggests that Hepatitis C virus (HCV) infection is associated with 
71 insulin resistance (IR) and Type 2 diabetes mellitus (T2DM (1-4). In

72 addition, several plausible pathways have been suggested to explain how

73 HCV influences IR and T2DM) (5-7). However, estimates of the size of

74 the effect of HCV on T2DM risk vary between different studies. Two

75 different meta-analyses of a total of 47 different studies both showed

76 approximately $70 \%$ increased odds/hazards of having diabetes for

77 individuals with HCV infection compared to individuals without HCV

78 infection (adjusted Odds Ratio (OR), 1.7; 95\% Confidence Interval (CI),

$79 \quad 1.2-2.5(3)$; and 1.7; 95\% CI, 1.2-2.2 (8)). A recent population based

80 cross sectional study from the US (9), however, found little evidence of

81 increased risk to test diabetes positive in people with, compared to

82 without, either current HCV-infection (OR, 1.1; 95\% CI, 0.6-1.9) or

83 with current or past HCV-infection (OR, 1.0; 95\% CI, 0.6-1.7). In

84 addition, a large population based cohort study from Southern Italy

85 showed that compared to $\mathrm{HCV}^{-v e}$ controls only people with $\mathrm{HCV}$ and

86 elevated alanine aminotransferase (ALT) levels were at higher odds of

87 developing T2DM (OR,1.5; 95\% CI, 1.0-2.2), while those with HCV

88 and normal baseline ALT levels were at lower odds (OR, 0.6; 95\% CI,

89 0.3-1.1) (10). Another study of people enrolled in a community-based

90 cohort in the US showed that HCV infection increased the risk of

91 developing diabetes (adjusted hazard ratio (HR), 11.6; 95\%CI 1.4-96.6), 
92 but only among those at high risk of diabetes (based on body mass index

93 and age) (11). Finally, a recent meta analysis suggested, on the basis of

94 limited evidence, that having diabetes can also be a risk factor for

95 contracting HCV (12).

96 The heterogeneity of findings from the different studies indicates

97 that, at a population level, the effect of HCV on T2DM risk is

98 comparably low and varies between different strata of the population.

99 Therefore, studies to estimate the size of the effect of HCV on T2DM in

100 the general population need to be sufficiently large to allow examination

101 of different strata of the population and need careful control of

102 confounding. Factors that increase the risk for diabetes and that might

103 differ between those with HCV and those without HCV include low

104 socioeconomic status $(13,14)$, a history of heroin dependence (15) and

105 methadone treatment (16), high alcohol consumption (17), smoking

106 (18), increasing age (19), male sex (19), non white ethnicity (20) and

107 higher body mass index (14).

108 To study the relationship between HCV infection and T2DM at a 109 population level, we compared the risk of T2DM diagnosis in all people

110 who have been diagnosed HCV antibody ${ }^{+v e}$ with the risk of T2DM

111 diagnosis in a three-times larger cohort of controls matched for the

112 major confounding factors of sex, neighbourhood and age. To ascertain 
113 wether any difference in the risk of T2DM was related to the virus itself

114 or to factors associated with HCV-infection, we compared the

115 relationship between HCV infection and T2DM in all people who had

$116{\text { tested (i) } \mathrm{HCV} \text { antibody }^{+v e} \text {, (ii) } \mathrm{HCV} \text { antibody }}^{+v e}$ and $\mathrm{RNA}^{+v e}$ and (iii)

$117 \mathrm{HCV}$ antibody $^{+v e}$ and $\mathrm{RNA}^{-v e}$. To reduce the potential effect of

118 ascertainment bias associated with being diagnosed for HCV infection,

119 we studied three different periods of T2DM diagnosis: (i) a diabetes

120 diagnosis at least 1 year prior to HCV diagnosis; (ii) within \pm 1 year of

121 HCV diagnosis and; (iii) later than one year post HCV diagnosis.

\section{Patients and Methods}

\section{Data sources for diagnosis of HCV and T2DM}

124 Scotland has comprehensive national disease databases of people 125 diagnosed with HCV-antibodies and of people diagnosed with diabetes.

126 The database of people diagnosed with HCV-antibodies held at Health

127 Protection Scotland holds information on more than 30,000 people from

128 all over Scotland who have tested HCV antibody ${ }^{+v e}$ between 1985 and

1292011 (see (21) for a description of the database). The Scottish Care

130 Information - Diabetes Collaboration (SCI-DC) manages a national

131 register that holds information on individuals with diagnosed diabetes 
132 (over 300,000) in Scotland and is estimated to have included over 99\%

133 of people with diagnosed diabetes since 2004. Individuals are included

134 on SCI-DC if they have a Read $\operatorname{code}^{1}$ for diabetes assigned in primary

135 care or if they are seen in a hospital diabetes clinic (for a description of

136 the database, see (22)).

\section{HCV antibody ${ }^{+v e}$ cohort}

138 For the period up to the end of 2011, 31,468 records of HCV antibody ${ }^{+v e}$

139 people from all over Scotland were held in the HCV diagnoses database.

140 From the database, information was extracted on forename initial, a

141 soundex encrypted version of the surname (soundex is a phonetic

142 algorithm for indexing names by sound, as pronounced in English), date

143 of birth, sex, RNA test results at first diagnosis (positive, negative,

144 unknown) and date of first $\mathrm{HCV}^{+v e}$ antibody test (hereafter referred to as

145 date of HCV diagnosis).

146 To enable linkage of the partially anonymised data in the HCV

147 database to other databases, 24,975 (79\%) records from the HCV

148 database were probabilistically linked to the database of the community

149 health index (CHI), a unique identifier used in medical records (23).

\footnotetext{
${ }^{1}$ Read codes are the standard clinical terminology system used in General Practice in the United Kingdom.
} 
150 After linkage, information from $\mathrm{CHI}$ was added to the $\mathrm{HCV}$ antibody ${ }^{+v e}$

151 cohort including full personal identifiers, postcode sector of residence at

152 the time of HCV diagnosis, an indicator for social deprivation of the area

153 of residence (Scottish Index of Multiple Deprivation, SIMD) (24) and an

154 indicator and date for migration from Scotland. We then excluded 107

155 people younger than 16 and a total of 588 individuals with missing or

156 unclear information on SIMD, sex and diagnosis date. After these

157 exclusions, 24,280 individuals remained in the study population (see

158 Figure 1 in the Appendix).

\section{HCV antibody ${ }^{-v e}$ cohort}

160 For every person in the HCV antibody ${ }^{+v e}$ cohort, up to three people were

161 randomly sampled without replacement from the CHI database who

162 were (i) born within one calendar year; (ii) of the same sex; (iii) alive at

163 the time of diagnosis of the matched person on the HCV database; (iv)

164 lived in the same postcode sector (but not in the same postcode) at the

165 time of HCV diagnosis; and (v) were not included in the HCV

166 antibody $^{+v e}$ cohort. Given the low prevalence of HCV in the Scottish

167 population (25), less than $2 \%$ of the HCV antibody ${ }^{-v e}$ cohort will likely

168 have undiagnosed HCV-infection; thus, this misclassification will have

169 negligible influence on the results. For 2118 people in the HCV 
170 antibody $^{+v e}$ cohort, no matching individual could be identified in the

171 CHI database; these people were excluded from the HCV antibody ${ }^{+v e}$

172 cohort. As a result, 22,162 matched groups were available for analysis.

173 People in the HCV antibody ${ }^{-v e}$ cohort were assigned an index date

174 which corresponded to the diagnosis date of their matched cohort 175 member.

\section{Diabetes}

177 To identify diagnosed diabetes status in both cohorts (HCV antibody ${ }^{+v e}$

178 and HCV antibody $^{-v e}$ ), data were deterministically linked to the 179 SCI-DC database based on CHI number. After linkage, information

180 from SCI-DC was added to the data including type of diabetes (T1DM,

181 T2DM and other/unknown) and date of diabetes diagnosis. For $11 \mathrm{HCV}$

182 antibody $^{+v e}$ people, diabetes was diagnosed but date of diabetes

183 diagnosis was not available; these individuals, together with their 31

184 matched individuals from the HCV antibody ${ }^{-v e}$ cohort, were removed 185 from analysis. An additional three individuals from the HCV 186 antibody $^{-v e}$ cohort with a diabetes diagnosis were removed as they had

187 no date for their diagnosis. A further $219 \mathrm{HCV}$ antibody ${ }^{+v e}$ individuals

188 were removed together with 652 matched individuals from the HCV 
189 antibody $^{-v e}$ cohort because they had been diagnosed with a type of

190 diabetes other than T2DM. Additionally, 451 people from the HCV

191 antibody $^{-v e}$ cohort were excluded because they had been diagnosed with

192 a type of diabetes other than T2DM.

\section{Morbidity and mortality}

194 To identify further censoring dates in both cohorts, data were then linked

195 deterministically to mortality data from the General Registrars Office of

196 Scotland (GRO, see (26) for a description of the database) and the date

197 of death was added to the cohort data. Cohort members were

198 additionally linked deterministically to hospital databases, to ascertain

199 whether, prior to the HCV-diagnosis date, they had been in hospital for

200 an alcohol-related admission (ICD9: 571.[0-3], 291.[0-9], 535.3, 425.5,

201 357.5, 305.0, 303.9; ICD10: E24.4, E51.2, F10.[0-9], G31.2, G62.1,

202 G72.1, I42.6, K29.2, K70.[0-9], K86.0, O35.4, P04.3, Q86.0, R78.0,

203 T51.[0,1,9], X[4,6]5, Y15, Y57.3, Y90.[3-8], Y91, Z50.2, Z71.4, Z72.1)

204 or for an obesity-related admission (ICD9: 278.[0-9]; ICD10: E66).

205 Three members of the HCV antibody ${ }^{+v e}$ cohort matched to two different

206 death records and were subsequently removed from analysis, leaving

20721,929 for analysis (Fig. 1). 


\section{Information Governance}

209 Data linkages were approved by the NHS National Services Scotland

210 Privacy Advisory Committee and use of the CHI database was approved

211 by the CHI Advisory Group. All linkages were undertaken at

212 Information Service Division, Scotland and all personal identifiable

213 information removed from the outputs prior to release of data to the

214 research team for analysis.

\section{Statistical analysis}

216 The probability of T2DM diagnosis for those in the HCV antibody ${ }^{+v e}$

217 compared to the HCV antibody ${ }^{-v e}$ cohort was determined for the

218 following three time periods: i) up-to one year before HCV diagnosis

219 (pre-HCV); ii) from one year before HCV diagnosis to one year after

$220 \mathrm{HCV}$ diagnosis (peri-HCV); and iii) from one year after HCV diagnosis

221 to the earlier of either the end of follow-up (November 1st, 2011), death,

222 migration out of Scotland or diagnosis of T2DM (post-HCV).

223 Generalized linear mixed models (R, package lme4) were used for

224 the analysis of the odds of T2DM diagnosis pre-HCV and peri-HCV.

225 Mixed effects Cox models (R, package coxme) were used for the

226 analysis of the hazard of T2DM diagnosis post-HCV. In all three

227 regression models, the year of $\mathrm{HCV}$ diagnosis (grouped into prior to 
2282000 and later than 1999), sex, social deprivation (grouped into three

229 groups using the original quintiles: $1-2=$ high, $3=$ medium and $4-5=$ low

230 deprivation) and age at HCV diagnosis were included as explanatory

231 variables. Fractional polynomials were used to model age at HCV

232 diagnosis (R, package mfp). To adjust for correlation within matched

233 groups, a random group effect was added to all three models.

234 To study if the estimated effect of HCV-infection on the probability

235 of T2DM diagnosis was modified by period of HCV diagnosis, sex,

236 social deprivation or age at HCV diagnosis, interaction-terms between

237 these variables and HCV were added to the full model. Likelihood ratio

238 tests were used for testing the statistical significance of interaction terms

239 and those interaction terms that were not statistically significant

$240(P>0.05)$ were removed. For statistically significant interaction terms, a

241 synergy index $(S)$ was calculated to demonstrate the excess risk from

242 exposure (to both exposures) when there is interaction relative to the risk

243 from exposure (to both exposures) without interaction. Influential

244 values, outliers and model fit were ascertained in the final models

245 excluding random group effects ( $R$, package boot). The assumption of

246 proportionality of hazards in the survival analysis was tested using

247 Schoenfeld residuals (R, package survival). 
248 To study the effect of chronic and resolved HCV infection, all final

249 models were re-run separately for those in the HCV antibody ${ }^{+v e}$ cohort

250 who were initially tested (i) RNA-positive (indicative of chronic HCV)

251 and (ii) RNA-negative (indicative of resolved HCV). Here, the HCV

252 antibody $^{-v e}$ cohorts were composed only of people who were matched

253 to RNA-positive (for ( i)) and RNA-negative (for (ii)) individuals.

\section{Results}

\section{Characteristics of the study population}

256 Table 1 shows the composition of the study population comprising

25721,929 people in the HCV antibody ${ }^{+v e}$ cohort and 65,074 people in the

258 matched HCV antibody ${ }^{-v e}$ cohort. Reflecting the composition of the

259 HCV antibody ${ }^{+v e}$ population in Scotland, people in the HCV

260 antibody $^{+v e}$ cohort were predominantly male (68\%), born between 1960

261 and 1980 (68\%), were diagnosed with HCV after the year 2000 (70\%)

262 and were living at the time of HCV diagnosis in areas of highest

263 deprivation (75\%). $72 \%$ of the people in the HCV antibody ${ }^{+v e}$ cohort

264 were $\mathrm{HCV}-\mathrm{RNA}^{+v e}, 18 \%$ were $\mathrm{HCV}^{-\mathrm{RNA}^{-v e}}$ and in $10 \%$ the RNA

265 status was unknown. More than $97 \%$ of people in the HCV antibody ${ }^{+v e}$ 
266 cohort could be matched to three HCV antibody ${ }^{-v e}$ people from the CHI

267 database, while for people born before 1950 fewer matches were 268 identified.

269 Median follow-up time from HCV-diagnosis to censoring or end of

270 follow-up was 6.4 years in the HCV antibody ${ }^{+v e}$ cohort and 6.6 years in

271 the HCV antibody $^{-v e}$ cohort; median age at HCV diagnosis was 33

272 years. During a total follow-up period of 151,020 person-years from

273 HCV-diagnosis to censoring in the HCV antibody ${ }^{+v e}$ cohort, 4016

274 people died (2.66 per 100 person-years). In the HCV antibody ${ }^{-v e}$ cohort, 275 the total follow-up period was 463,977 person-years with 2633 deaths 276 recorded (0.57 per 100 person-years). The proportion of people who

277 have had an alcohol-related hospitalization prior to HCV-diagnosis was

278 considerably higher in the HCV antibody ${ }^{+v e}$ cohort (22\%) than in the

279 HCV antibody ${ }^{-v e}$ cohort (4.5\%), while there was not much difference in

280 the proportion of people who have had an obesity-related hospitalization 281 (both 0.3\%) prior to HCV-diagnosis. 


\section{Diagnosis of T2DM in the HCV antibody ${ }^{+v e}$ cohort compared to the}

\section{HCV antibody ${ }^{-v e}$ cohort}

284 Of 21,929 people in the HCV antibody ${ }^{+v e}$ cohort, 628 (2.86\%) had been

285 diagnosed with T2DM, of whom 187 (30\%) had been diagnosed with

286 T2DM more than a year before they had been diagnosed HCV-positive

287 and 141 (22\%) had been diagnosed with T2DM within one calender year

288 of their HCV diagnosis (Table 2). This compares to 1772 out of 65,074

289 (2.72\%) in the HCV antibody ${ }^{-v e}$ cohort who have been diagnosed with

290 T2DM, of whom 524 (30\%) had been diagnosed with T2DM more than

291 a year before the matched person in the HCV antibody ${ }^{+v e}$ cohort had

292 been diagnosed HCV-positive and 184 (10\%) had been diagnosed with

293 T2DM within one calender year of their HCV diagnosis (Table 2). The

294 difference between both cohorts in the proportion of people who were

295 diagnosed with T2DM (0.14\%) indicates an excess of 32 cases in HCV

296 antibody $^{+v e}$ study population or 14 per 10,000 HCV-infected people,

297 while for those who tested $\mathrm{RNA}^{+v e}$ and $\mathrm{RNA}^{-v e}$, excess risks of 34 and

29820 per 10,000, respectively, were found. In both HCV antibody ${ }^{+v e}$ and

299 HCV antibody ${ }^{-v e}$ cohorts the median age at diagnosis with T2DM was $300 \quad 45$ years. 
301 Odds of T2DM diagnosis up to one year prior to HCV diagnosis

302 In the HCV antibody ${ }^{-v e}$ cohort, male sex and high social deprivation

303 were associated with increased risks of having a diagnosis of T2DM in

304 the period up to one year prior to HCV diagnosis. However, in the HCV

305 antibody $^{+v e}$ cohort, the same variables were associated with decreased

306 risk (Table 3). The 4345 women in the HCV antibody ${ }^{-v e}$ cohort who

307 resided in areas of lowest deprivation had the lowest risk of having a

308 diagnosis of T2DM (0.4\%), while the 941 women in the HCV

309 antibody $^{+v e}$ cohort who resided in areas of lowest deprivation had the

310 highest risk (2.4\%; OR, 4.02; 95\% CI, 2.29-7.04 $P<0.01$ ). The 28,267

311 men in the HCV antibody ${ }^{-v e}$ cohort who resided in areas of highest

312 deprivation had a higher risk of having a diagnosis of T2DM (0.9\%) than

313 the 11,131 men in the HCV antibody ${ }^{+v e}$ cohort who resided in areas with

314 the same high deprivation (0.5\%; OR, 0.61; 95\% CI, 0.43-0.87 $P<0.01$ ).

315 The synergy indices show negative interaction on an additive scale,

316 indicating that the combined effects of male sex and HCV-infection and

317 deprivation and $\mathrm{HCV}$-infection were less than the sum of the effects of

318 male sex and HCV-infection and deprivation and HCV-infection.

319 Similar ORs were estimated when restricting the HCV-positive

320 cohort to either only people who have tested $\mathrm{RNA}^{+v e}$ (indicative of 
321 chronic infection) or those who have tested RNA-negative (indicative of 322 past infection; Table 3).

\section{Odds of T2DM diagnosis within \pm one year of HCV diagnosis}

324 In the HCV antibody ${ }^{-v e}$ cohort, male sex was associated with increased

325 risks of having a diagnosis of T2DM in the period within one year of

326 HCV diagnosis. However, in the HCV antibody ${ }^{+v e}$ cohort, there was

327 little difference between men and women (Table 4). The lowest risk of

328 having a diagnosis of T2DM was observed for the 20,626 women in the

329 HCV antibody ${ }^{-v e}$ cohort (0.2\%) while the highest risk was observed for

330 the 6996 women in the HCV antibody ${ }^{+v e}$ cohort $(0.7 \%$; OR, 3.78; $95 \%$

331 CI, 2.29-6.24 $P<0.01$ ). Increased risks of having a diagnosis of T2DM

332 were also observed in the 14,746 men in the HCV antibody ${ }^{+v e}$ cohort

333 (0.6\%) compared to men in the HCV antibody ${ }^{-v e}$ cohort $(0.3 \%)$, but

334 because of the increased risk in males in the $\mathrm{HCV}$ antibody ${ }^{-v e}$ cohort,

335 the estimated adjusted OR was lower than in women (OR, 1.97; 95\% CI,

336 1.46-2.65; $P<0.01)$. Again, the synergy index indicates negative

337 interaction on an additive scale between the effect of male sex and

338 HCV-infection $(S=0.71)$. 
339 The estimated increased odds for women in the HCV antibody ${ }^{+v e}$

340 cohort compared to those in the HCV antibody ${ }^{-v e}$ cohort further

341 increased when only women were included in the data set who had tested

342 RNA-positive (OR, 4.57). Increased odds were also calculated for those

343 women who tested RNA-negative (OR, 2.89). For men, estimates for the

344 effect of HCV-infection on the odds of having a diagnosis of T2DM

345 were similar in the full data set (OR, 1.97), the RNA-positives (OR,

346 2.07) or RNA-negatives (OR, 2.02). However, restricting the cohort to

347 RNA-negatives, the variance for estimates increased and some of the

348 differences in the odds between people in the HCV-positive cohort and

349 the HCV antibody ${ }^{-v e}$ cohort were not statistically significant (Table 4).

350 Hazard of T2DM diagnosis later than one year after HCV diagnosis

351 In the HCV antibody $^{-v e}$ cohort, increasing social deprivation was

352 associated with an increased hazard of having a diagnosis of T2DM in

353 the period later than one year after HCV diagnosis. However, in the

354 HCV antibody ${ }^{+v e}$ cohort, increasing social deprivation was associated

355 with a decreased hazard of having a diagnosis of T2DM (Table 5). The

356 lowest hazard of having a diagnosis of T2DM was observed for the

35714,298 people in the HCV antibody $^{+v e}$ cohort who lived in areas of 
358 highest deprivation (1.4\%) which was (non-significantly) lower than the

359 hazard for the 34,470 members of the HCV antibody ${ }^{-v e}$ cohort living in

360 the same areas of high deprivation (1.9\%; HR, 0.88; 95\% CI, 0.75-1.03

$361 P=0.11)$. The highest hazard was observed for the 2401 people in the

362 HCV antibody ${ }^{+v e}$ cohort who lived in areas of lowest deprivation (2.5\%)

363 which was (significantly) higher than the hazard for the 10,957 members

364 of the HCV antibody ${ }^{-v e}$ cohort living in the same areas of low

365 deprivation (1.6\%; HR, 1.53; 95\% CI, 1.14-2.04 $P<0.01)$. The synergy

366 indices indicate negative interaction on an additive scale between the

367 effect of deprivation and HCV-infection.

368 Slightly higher effects of HCV-infection on the hazard of being

369 diagnosed with T2DM more than one year after HCV diagnosis were

370 estimated when restricting the HCV-positive cohort to those who have

371 tested RNA $^{+v e}$ (indicative of chronic infection). Increased hazards were

372 also estimated for those HCV antibody ${ }^{+}{ }^{2 e}$ who tested RNA-negative and

373 who lived in areas with high or low deprivation; however, due to the

374 small sample size, those differences were not statistically significant

375 (Table 5). 


\section{Discussion}

377 This study compares the risk of receiving a diagnosis of T2DM in a

378 cohort of all people who have been diagnosed HCV antibody ${ }^{+v e}$ in

379 Scotland (the vast majority of whom will have acquired infection

380 through injecting drug use) with that of a three times larger HCV

381 antibody $^{-v e}$ cohort matched on year of birth, sex and neighbourhood.

382 The HCV antibody ${ }^{+v e}$ cohort was further stratified by RNA-status to

383 check whether any additional risk attributed to HCV infection was

384 related to the virus infection itself or to other factors related to the

385 infection. Further the effect of HCV infection in three time periods -

386 pre-HCV, peri-HCV and post-HCV diagnosis was studied to investigate

387 any bias due to increased testing for T2DM at the time of HCV

388 diagnosis.

389 This study shows that nationwide over a time-period of

390 approximately 12 years there were approximately 14 additional cases of

391 T2DM for every 10,000 HCV-infected people compared to what would

392 have been observed in a HCV antibody ${ }^{-v e}$ cohort of identical size and

393 characteristic. The excess risk was similarly low among RNA ${ }^{+v e}$ when

394 taking into account the excess risk among $\mathrm{RNA}^{-v e}$. Including those with

395 HCV who are undiagnosed (nationwide approximately 50\%, (25) ), we 
396 would expect that the total excess number of people with HCV-antibody

397 infection who have developed HCV-related T2DM up to this point in 398 time is less than 100.

399 While this is the first study to estimate the extra number of

400 HCV-related T2DM cases for a whole nation, increases in risk of those

401 with HCV have been reported elsewhere (1-4). For the national health

402 system of Scotland, compared to total number of people reported to have

403 been diagnosed with T2DM (265,000 between 2000 and 2012), the

404 increase of less than 100 cases in a 12-year period is relatively small.

405 Similarly, for the HCV-infected individual, compared to lifestyle

406 choices related to an increase in T2DM risk, the increase in risk related

407 to HCV-infection from $2.7 \%$ to $2.9 \%$ seems comparably low. The

408 relatively small difference in risk observed in our study indicates the

409 necessity to study the association between HCV-infection and T2DM in

410 large, well-defined study populations. Ruhl et al. (9) found no

411 association between HCV and either diabetes or insulin resistance (IR)

412 in their US population based study, involving $277 \mathrm{HCV}$ antibody $^{+v e}$

413 individuals (compared to the 21,929 studied here); a relationship

414 between $\mathrm{HCV}$ and diabetes could only be found among those with

415 elevated enzyme activity. Ruhl et al. thus suggest that the previously

416 reported findings of a strong relationship with diabetes may have 
417 resulted from the increased liver enzyme activity in the HCV

418 populations studied (9). Further, a recent meta-analysis has found an 419 association between the presence of IR and advanced fibrosis in those 420 with HCV genotype 1 (the most common genotype in the US), but not

421 for genotype 3 (27). We lacked data on liver enzyme activity, IR and 422 HCV genotype in this database linkage study to be able to investigate 423 this further in a larger cohort.

424 Matching allowed us to control for the effects of age, sex and 425 neighbourhood; the latter being a proxy for social deprivation and 426 regional differences in testing and recording for both conditions. 427 However, estimates of the number of additional cases of T2DM in those 428 with HCV-infection could have been biased from other risk factors for 429 T2DM for which information was not available. Ethnicity is known to be 430 related to T2DM, with people of South Asian background living in the 431 UK having 3-4 times higher risk of developing T2D during their life 432 compared to the majority white population (20). Moreover, people of 433 South Asian ethnicity are known to have a higher prevalence of HCV 434 (28), so a higher proportion of people with South Asian ethnicity would 435 be expected in the HCV-positive cohort. However, the South Asian 436 population in Scotland is very small ( $\approx 1 \%$ in the 2001 census $)$, so that 437 confounding from a varying ethnic composition of the HCV-positive 
438 cohort and the HCV-negative cohort can be expected to be small.

439 Body-mass is a further known risk factor for T2DM, and it is possible

440 that differences in BMI may confound the association between

441 diagnoses of HCV and T2DM. However, since social deprivation and

442 obesity are closely correlated in Scotland (14), matching by

443 neighbourhood should have increased comparability of both cohorts, as

444 indicated by similar proportions of people with a record of an obesity

445 related hospitalization in the $\mathrm{HCV}$ antibody ${ }^{+v e}$ and the $\mathrm{HCV}$ antibody ${ }^{-v e}$

446 cohort. Similarly, alcohol consumption is a known risk factor for T2DM

447 (29) and because alcohol consumption is positively related to

448 HCV-status it could be expected that the proportion of people with high

449 alcohol consumption was higher in the HCV-positive cohort compared

450 to the HCV-negative cohort. Indeed, compared to people in the

451 HCV-negative cohort, people in the HCV-positive cohort had a

452 4.6-times higher risk of having an alcohol-related hospitalization. This

453 bias from other risk factors related to T2DM might explain the 454 observation in our study that compared to people in the HCV 455 antibody $^{-v e}$ cohort, people with resolved HCV-infection 456 (RNA-negative) were still at higher risk of having a diagnosis of T2DM. 457 The study also shows that the effect of diagnosed HCV-infection on 458 the relative proportions of people with a diagnosis of T2DM was time 
459 dependent. Partitioning of the risk period clearly showed that the

460 increased risk is mainly due to increased T2DM diagnosis around the

461 time of HCV diagnosis, while the $10 \%$ increased risk more than one year

462 prior to HCV diagnosis and one year post HCV diagnosis were

463 considerably lower than the estimate from the meta-analyses.

464 Interestingly, the estimate of a $10 \%$ increased relative risk is very similar

465 to that from the largest cohort study that had been included in the

466 meta-analyses (30) although the estimate of absolute T2DM prevalence

467 in the HCV antibody ${ }^{-v e}$ cohort in our study (3.2\%) was much lower than

468 that in the US study (13\%) or indeed any other cohort study but one

469 included in the meta-analyses. Increased T2DM within \pm 1 year is likely

470 related to ascertainment bias. However, neither guidelines by the

471 Scottish Intercollegiate Guideline Network (SIGN guidelines 116) nor

472 by the National Institute of Clinical Excellence recommend testing for

473 HCV infection in people diagnosed with T2DM and guidelines by the

474 European Association for the Study of the Liver only recommend testing

475 for T2DM prior to treatment for HCV infection, since 'poorly controlled

476 diabetes' is a contra-indication for treatment with interferon containing

477 regimens. Therefore, the most likely reason for the increased T2DM

478 diagnosis peri-HCV diagnosis is related to people showing clinical

479 symptoms indicative of liver disease. It seems likely that for people with 
480 signs of liver disease, a blood sample for glucose testing is collected at 481 the same time as samples for $\mathrm{HCV}$ tests and liver function 482 measurements. We do not have access to laboratory test databases in 483 order to investigate the potential for ascertainment bias further. While 484 there was a highly significant correlation between increasing age and the 485 risk of T2DM diagnosis, there was no significant increase with age in the 486 effect of HCV infection on the risk of T2DM ( $P=0.34$ for inclusion of an $487 \mathrm{HCV}^{*}$ age interaction term). This result indicates that the observed effect 488 of HCV infection on the risk of T2DM is more likely caused by other 489 factors related to HCV infection than by the (slowly progressing) action 490 of the virus. However, our HCV infected cohort is still relatively young 491 (median age at HCV diagnosis was 33 years) and has been followed up 492 for a relatively short time (median of 6.4 years), thus the excess risk of 493 T2DM may still change as our cohort advances in age and duration of 494 infection.

$495 \quad$ Male sex and living in areas of highest deprivation decreased effects 496 of HCV infection on the risk of T2DM diagnosis. This effect 497 modification was not related to follow-up time, age at HCV-infection or 498 RNA-status since those did not differ within sex and social deprivation. 499 Since male sex and high deprivation are positively related to T2DM risk, 500 our observation does not confirm the suggestion from (11) that relative 
501 effects of HCV on T2DM risk are higher in people at increased risk of

502 T2DM. However, the effect modification could be explained by 503 different uptake of health care (and thereby testing for diabetes) in men

504 living in areas of high deprivation. The effect modification could explain

505 some of the heterogeneity that both meta analyses found, since few of

506 the reviewed studies stratified by sex and none by social deprivation.

507 However, widely accepted biological models of the effects of HCV

508 infection on T2DM risk (5-7) do not explain the observed effect

509 modification. Moreover, while sex, social deprivation and year of birth

510 were included in our matched analysis to increase efficiency of the study

511 (31), the analysis of effect modification by sex, social deprivation, year

512 and age was purely exploratory.

513 Ideally, every person in the HCV-positive cohort should have been

514 followed-up from the date of HCV-infection to development of T2DM

515 or censoring. However, because date of HCV-infection was unknown,

516 the follow-up period and thereby the risk of T2DM diagnosis pre-HCV

517 diagnosis was heterogeneous. Additionally, the T2DM database is only

518 approximately complete from 2004 onwards, with regional differences

519 in the date from which diagnoses of T2DM were reported to the

520 database. By matching people in the $\mathrm{HCV}$ antibody $^{-v e}$ cohort to those in

521 the HCV antibody ${ }^{+v e}$ cohort by year of birth and place of residence and 
522 by adequately controlling for the effect of matching in the analysis we 523 managed to reduce the potential bias for the odds ratio from 524 heterogeneous follow-up times. However, the estimated odds of T2DM

525 diagnosis pre-HCV diagnosis in both cohorts are difficult to interpret. In 526 addition, since date of HCV-infection and date of onset of T2DM both

527 were unknown to us, the temporal relationship of onset of HCV infection

528 and T2DM is not known. Indeed, T2DM has been described as a risk 529 factor for contracting $\mathrm{HCV}$ (12). However, an estimated $86 \%$ of

530 HCV-infection in Scotland is related to injecting drug use (32) and a 531 large fraction of those diagnosed HCV-positive will have been infected 532 in their early drug using career. Given that the risk of developing T2DM 533 increases with age, it is unlikely that the increased risk for HCV in those 534 with T2DM was responsible for the results of our study.

535 Our study has demonstrated that on the population level the size of 536 the effect of HCV antibody status on T2DM is smaller than effects of 537 many life style choices (e.g., obesity, smoking and alcohol consumption) 538 and therefore not as significant a public health concern as previously 539 suggested from predominantly clinic based studies. Findings were

540 similarly observed for both $\mathrm{RNA}^{+ \text {ves }}$ (chronic) and $\mathrm{RNA}^{-v e s}$ (resolved)

541 which further indicates that the observed differences in risk of T2DM

542 diagnosis were not related to the virus itself but to factors related to the 
543 infection (e.g., factors related to drug abuse). However, given the

544 increased risk for HCV-related disease progression in those affected by

545 both conditions (33), further research is required to identify whether

546 screening and earlier treatment for T2DM improves outcomes among

547 people with a diagnosis of chronic HCV. Socio-economic status, sex and

548 a history of alcohol use and injecting drug use modify the effect of HCV

549 on T2DM which could explain some of the discrepancies between

550 different studies given the different patterns of these factors in different

551 populations.

\section{Acknowledgement}

553 We are grateful to the following virologists for their support with the

554 HCV diagnosis database: Dr Kate Templeton (East of Scotland

555 Specialist Virology Centre, Royal Infirmary of Edinburgh, Edinburgh),

556 Dr Celia Aitkin (West of Scotland Specialist Virology Centre, Gartnavel

557 General Hospital, Glasgow), Dr Paul McIntyre (Department of Medical

558 Microbiology, Ninewells Hospital and Medical School, Dundee), and Dr

559 Pamela Molyneaux (Department of Medical Microbiology, University

560 Medical School, Foresterhill, Aberdeen). 


\section{Literature}

563 (1) Arao M, Murase K, Kusakabe A, Yoshioka K, Fukuzawa Y,

564 Ishikawa T, et al. Prevalence of diabetes mellitus in Japanese patients

565 infected chronically with hepatitis C virus. J Gastroenterol

$566 \quad 2003 ; 38(4): 355-360$.

567 (2) Serfaty L, Capeau J. Hepatitis C, insulin resistance and diabetes:

568 clinical and pathogenic data. Liver Int 2009 Mar;29 Suppl 2:13-25.

569 (3) Naing C, Mak JW, Ahmed SI, Maung M. Relationship between

570 hepatitis $\mathrm{C}$ virus infection and type 2 diabetes mellitus: meta-analysis.

571 World J Gastroenterol 2012 Apr 14;18(14):1642-1651.

572 (4) Dai CY, Yeh ML, Huang CF, Hou CH, Hsieh MY, Huang JF, et al.

573 Chronic hepatitis C infection is associated with insulin resistance and

574 lipid profiles. J Gastroenterol Hepatol 2013 Jun 28. Epub ahead of print.

575 (5) Bose SK, Ray R. Hepatitis C virus infection and insulin resistance.

576 World J Diabetes 2014 Feb 15;5(1):52-58.

577 (6) Alexander GJ. An association between hepatitis C virus infection and

578 type 2 diabetes mellitus: what is the connection? Ann Intern Med 2000

579 Oct 17;133(8):650-652. 
580 (7) Naing C, Mak JW, Wai N, Maung M. Diabetes and

581 infections-hepatitis C: is there type 2 diabetes excess in hepatitis C

582 infection? Curr Diab Rep 2013 Jun;13(3):428-434.

583 (8) White DL, Ratziu V, El-Serag HB. Hepatitis C infection and risk of

584 diabetes: a systematic review and meta-analysis. J Hepatol 2008

585 Nov;49(5):831-844.

586 (9) Ruhl CE, Menke A, Cowie CC, Everhart JE. Relationship of

587 Hepatitis C Virus Infection with Diabetes in the U.S. Population.

588 Hepatology 2014 Oct; 60 (4):1139-1149.

589 (10) Montenegro L, De Michina A, Misciagna G, Guerra V, Di Leo A.

590 Virus C hepatitis and type 2 diabetes: a cohort study in southern Italy.

591 Am J Gastroenterol 2013 Jul;108(7):1108-1111.

592 (11) Mehta SH, Brancati FL, Strathdee SA, Pankow JS, Netski D,

593 Coresh J, et al. Hepatitis C virus infection and incident type 2 diabetes.

594 Hepatology 2003 Jul;38(1):50-56.

595 (12) Guo X, Jin M, Yang M, Li J. Type 2 diabetes mellitus and the risk

596 of hepatitis c virus infection: a systematic review. Scientific Reports

597 2013;3(2981):1-8.

598 (13) Espelt A, Borrell C, Roskam AJ, Rodriguez-Sanz M, Stirbu I,

599 Dalmau-Bueno A, et al. Socioeconomic inequalities in diabetes mellitus 
600 across Europe at the beginning of the 21st century. Diabetologia 2008

601 Nov;51(11):1971-1979.

602 (14) Evans JM, Newton RW, Ruta DA, MacDonald TM, Morris AD.

603 Socio-economic status, obesity and prevalence of Type 1 and Type 2

604 diabetes mellitus. Diabet Med 2000 Jun;17(6):478-480.

605 (15) Pereska Z, Bozinovska C, Dimitrovski C, Cakalarovski K,

606 Chibishev A, Zdravkovska M, et al. Heroin dependence duration

607 influences the metabolic parameters: mechanisms and consequences of

608 impaired insulin sensitivity in hepatitis $C$ virus seronegative heroin

609 dependents. J Addict Med 2012 Dec;6(4):304-310.

610 (16) Fareed A. Predictors of diabetes mellitus and abnormal blood

611 glucose in patients receiving opioid maintenance treatment. American

612 journal on addictions 2013;22(4):411.

613 (17) Beulens JW. Estimating the mediating effect of different

614 biomarkers on the relation of alcohol consumption with the risk of type 2

615 diabetes. Ann Epidemiol 2013;23(4):193-197.

616 (18) Kim SJ, Jee SH, Nam JM, Cho WH, Kim JH, Park EC. Do early

617 onset and pack-years of smoking increase risk of type II diabetes? BMC

618 Public Health 2014 Feb 19;14(1):178. 
619 (19) Wild S, Roglic G, Green A, Sicree R, King H. Global prevalence of 620 diabetes: estimates for the year 2000 and projections for 2030. Diabetes 621 Care 2004 May;27(5):1047-1053.

622 (20) Fischbacher CM, Bhopal R, Steiner M, Morris AD, Chalmers J. Is

623 there equity of service delivery and intermediate outcomes in South

624 Asians with type 2 diabetes? Analysis of DARTS database and summary

625 of UK publications. J Public Health (Oxf) 2009 Jun;31(2):239-249.

626 (21) Shaw L, Taylor A, Roy K, Cameron S, Burns S, Molyneaux P, et al.

627 Establishment of a database of diagnosed HCV-infected persons in

628 Scotland. Communicable Disease and Public Health / PHLS

629 2003;6(4):305-310.

630 (22) Scottish Diabetes Group. Diabetes in Scotland. Available at:

631 http://www.diabetesinscotland.org.uk, 2014.

632 (23) NHS National Services Scotland. What is the Community Health 633 Index (CHI)? Available at:

634 http://www.shsc.scot.nhs.uk/upload/file/national_committee_services/c

635 hiag/2010_10_19_what_is_the_community_health_index.pdf, 2014.

636 (24) Donnan PT, Leese GP, Morris AD, Diabetes Audit and Research in

637 Tayside, Scotland/Medicine Monitoring Unit Collaboration.

638 Hospitalizations for people with type 1 and type 2 diabetes compared 
639 with the nondiabetic population of Tayside, Scotland: a retrospective

640 cohort study of resource use. Diabetes Care 2000

641 Dec;23(12):1774-1779.

642 (25) Harris H, editors. Hepatitis C in the UK: Annual Report 2014.

643 Available at:

644 https://www.gov.uk/government/uploads/system/uploads/attachment_d

645 ata/file/337115/HCV_in_the_UK_2014_24_July.pdf; 2014.

646 (26) General Register Office for Scotland. Vital events: Technical

647 Report. Available at:

648 http://www.gro-scotland.gov.uk//statistics//theme//vital-events//deaths//

649 index.html. (Accessed: April 2014).

650 (27) Patel S, Jinjuvadia R, Patel R, Liangpunsakul S. Insulin resistance

651 is associated with significant liver fibrosis in chronic hepatitis C

652 patients: a systemic review and meta-analysis. J Clin Gastroenterol.

6532016 Jan; 50(1): 80-4.

654 (28) Uddin G, Shoeb D, Solaiman S, Marley R, Gore C, Ramsay M, et

655 al. Prevalence of chronic viral hepatitis in people of south Asian

656 ethnicity living in England: the prevalence cannot necessarily be

657 predicted from the prevalence in the country of origin. J Viral Hepat

$658 \quad$ 2010;17(5):327-35. 
659 (29) Wannamethee SG, Shaper AG, Perry IJ, Alberti KG. Alcohol

660 consumption and the incidence of type II diabetes. J Epidemiol

661 Community Health 2002 Jul;56(7):542-548.

662 (30) Butt AA, Evans R, Skanderson M, Shakil AO. Comorbid medical

663 and psychiatric conditions and substance abuse in HCV infected persons

664 on dialysis. J Hepatol 2006 May;44(5):864-868.

665 (31) Greenland S, Morgenstern H. Matching and efficiency in cohort

666 studies. Am J Epidemiol 1990 Jan;131(1):151-159.

667 (32) McDonald SA, Hutchinson SJ, Schnier C, McLeod A, Goldberg

668 DJ. Estimating the number of injecting drug users in Scotland's

669 HCV-diagnosed population using capture-recapture methods. Epidemiol

670 Infect 2014 Jan;142(1):200-207.

671 (33) Arase Y, Kobayashi M, Suzuki F, Suzuki Y, Kawamura Y, Akuta

$672 \mathrm{~N}$, et al. Effect of type 2 diabetes on risk for malignancies includes

673 hepatocellular carcinoma in chronic hepatitis C. Hepatology 2013

674 Mar;57(3):964-973.

675 
677 Table 1: Characteristics of the study population

678

\begin{tabular}{|c|c|c|c|c|c|}
\hline Variable & Level & $\begin{array}{l}\mathrm{HCV} \mathrm{Ab}^{+v e} \\
\text { cohort }(\mathrm{N})\end{array}$ & $\begin{array}{l}\text { HCV Ab }{ }^{-v e} \\
\text { cohort }(\mathrm{N})\end{array}$ & $\%$ & $\begin{array}{r}\% \\
\text { Complete } \\
\text { Matches }{ }^{1}\end{array}$ \\
\hline \multirow[t]{2}{*}{ Sex } & Women & 7067 & 20,956 & 32 & 97 \\
\hline & Men & 14,862 & 44,118 & 68 & 97 \\
\hline \multirow[t]{5}{*}{ Year of birth } & $<1950$ & 1335 & 3859 & 6 & 90 \\
\hline & 1950-1959 & 2876 & 8521 & 13 & 96 \\
\hline & 1960-1969 & 7246 & 21,545 & 33 & 97 \\
\hline & 1970-1979 & 7616 & 22,656 & 35 & 98 \\
\hline & $\geq 1980$ & 2856 & 8493 & 13 & 97 \\
\hline \multirow[t]{2}{*}{ Year of diagnosis } & $<2000$ & 6592 & 19526 & 30 & 96 \\
\hline & $\geq 2000$ & 15,337 & 45,548 & 70 & 97 \\
\hline \multirow[t]{3}{*}{ Deprivation } & Low & 2824 & 13,604 & $13 / 21^{3}$ & 96 \\
\hline & Medium & 2678 & 9628 & $12 / 15^{3}$ & 96 \\
\hline & High & 16,427 & 41,842 & $75 / 64^{3}$ & 97 \\
\hline $\begin{array}{l}\text { Alcohol-related } \\
\text { hospitalization }\end{array}$ & Yes & 4812 & 2942 & $22 / 4.5^{3}$ & \\
\hline $\begin{array}{l}\text { Obesity-related } \\
\text { hospitalization }^{2} \\
\end{array}$ & Yes & 60 & 209 & $0.3 / 0.3^{3}$ & \\
\hline Total & & 21,929 & 65,074 & & 97 \\
\hline
\end{tabular}

${ }^{1} \mathrm{~A}$ complete match is 1 person in the HCV antibody ${ }^{+v e}$ cohort and 3 people in the HCV antibody ${ }^{-v e}$ cohort matched on year of birth, sex and postcode sector of residence.

${ }^{2}$ Alcohol and obesity related hospitalization prior to HCV diagnosis; ICD9 codes and ICD10 codes as listed in patients and methods.

${ }^{3} \mathrm{HCV}$ antibody ${ }^{+v e}$ and HCV antibody ${ }^{-v e}$, respectively. 
681 Table 2: Number (and proportion) of people with T2DM in the HCV antibody ${ }^{+v e}$ 682 cohort (including for those $\mathrm{PCR}^{+\mathrm{ve}}$ and $\mathrm{PCR}^{-\mathrm{ve}}$ ) and in the $\mathrm{HCV}$ antibody ${ }^{-v e}$ cohort 683 according to time since HCV diagnosis.

\begin{tabular}{|c|c|c|c|c|c|c|c|c|}
\hline \multirow{2}{*}{$\begin{array}{l}\text { Period since } \\
\text { HCV } \\
\text { diagnosis }^{1}\end{array}$} & \multicolumn{2}{|c|}{$\begin{array}{l}\mathrm{HCV} \mathrm{Ab}{ }^{-v e} \\
(\mathrm{~N}=65,074)\end{array}$} & \multicolumn{2}{|c|}{$\begin{array}{l}\text { HCV Ab }{ }^{+v e} \\
(\mathrm{~N}=21,929)\end{array}$} & \multicolumn{2}{|c|}{$\begin{array}{c}\text { HCV Ab }{ }^{+v e} \& \text { PCR }^{+v e} \\
(N=15,827)\end{array}$} & \multicolumn{2}{|c|}{$\begin{array}{c}\text { HCV Ab }{ }^{+v e} \& \\
\operatorname{PCR}^{-v e}(N=3,927)\end{array}$} \\
\hline & Diabetes $^{+\mathrm{ve}}$ & $\%$ & Diabetes $^{+\mathrm{ve}}$ & $\%$ & Diabetes $^{+\mathrm{ve}}$ & $\%$ & Diabetes $^{+\mathrm{ve}}$ & $\%$ \\
\hline$>1$ year pre & 524 & 0.81 & 187 & 0.85 & 157 & 0.99 & 23 & 0.59 \\
\hline \pm 1 year & 184 & 0.28 & 141 & 0.64 & 115 & 0.73 & 18 & 0.46 \\
\hline$>1$ year post & 1064 & 1.64 & 300 & 1.37 & 234 & 1.48 & 49 & 1.25 \\
\hline Total & 1772 & 2.72 & 628 & 2.86 & 506 & 3.20 & 90 & 2.29 \\
\hline
\end{tabular}

$684{ }^{1}$ For those in the HCV antibody ${ }^{-v e}$ cohort, HCV diagnosis data was taken to be the 685 same as their respective HCV antibody ${ }^{+v e}$ cohort members, for the purpose of analysis.

686

687

688

689 
691 Table 3: Odds of having been diagnosed with T2DM in the period up to 1 year before

692 HCV diagnosis in the HCV antibody ${ }^{+v e}$ cohort (total and broken down by PCR status)

693 compared to the HCV antibody ${ }^{-v e}$ cohort $^{1,2}$

694

\begin{tabular}{|c|c|c|c|c|}
\hline Sex & Deprivation & $\begin{array}{l}\text { Diabetes }^{+v e} \\
/ \mathrm{HCV} \mathrm{Ab}^{-v e}\end{array}$ & $\begin{array}{l}\text { Diabetes }^{+v e} \\
/ \mathrm{HCV} \mathrm{Ab}^{+v e}\end{array}$ & $\begin{array}{rr}\mathrm{aOR}^{3} & \mathrm{~S}^{4} \\
(95 \% \mathrm{CI} ; P) & \end{array}$ \\
\hline \multicolumn{5}{|c|}{ Antibody ${ }^{+v e}$} \\
\hline $\mathrm{F}$ & Low & $17 / 4345$ (0.4\%) & 23/941 (2.4\%) & 4.02 (2.32-6.96); $P<0.01$ \\
\hline $\mathrm{F}$ & Medium & 16/3036 (0.5\%) & $10 / 830(1.2 \%)$ & $1.92(0.95-3.86) ; P=0.080 .42$ \\
\hline $\mathrm{F}$ & High & $101 / 13,575(0.7 \%)$ & $38 / 5296(0.7 \%)$ & 1.05 (0.66-1.69); $P=1.000 .32$ \\
\hline M & Low & 77/9259 (0.8\%) & 40/1883 (2.1\%) & 2.33 (1.42-3.83); $P<0.010 .62$ \\
\hline M & Medium & $57 / 6592(0.9 \%)$ & 19/1848 (1.0\%) & $1.11(0.58-2.11) ; P=0.990 .28$ \\
\hline M & High & $256 / 28,267(0.9 \%)$ & $57 / 11,131(0.5 \%)$ & $0.61(0.43-0.87) ; P<0.010 .15$ \\
\hline \multicolumn{5}{|c|}{ Antibody $^{+v e}$ and $\mathrm{PCR}^{+v e}$} \\
\hline $\mathrm{F}$ & Low & 12/3067 (0.4\%) & 18/661 (2.7\%) & 4.35 (2.33-8.13); $P<0.01$ \\
\hline $\mathrm{F}$ & Medium & 10/2117 (0.5\%) & 7/575 (1.2\%) & 2.05 (0.93-4.50); $P=0.090 .42$ \\
\hline $\mathrm{F}$ & High & 80/9098 (0.9\%) & $33 / 3576(0.9 \%)$ & 1.14 (0.67-1.93); $P=0.960 .35$ \\
\hline M & Low & $59 / 6886(0.9 \%)$ & $34 / 1375$ (2.5\%) & 2.61 (1.50-4.55); $P<0.010 .63$ \\
\hline M & Medium & $44 / 4877(0.9 \%)$ & $16 / 1360(1.2 \%)$ & $1.23(0.60-2.54) ; P=0.930 .30$ \\
\hline $\mathrm{M}$ & High & $202 / 20,936(1.0 \%)$ & 49/8280 (0.6\%) & $0.68(0.46-1.01) ; P=0.060 .19$ \\
\hline \multicolumn{5}{|c|}{ Antibody $^{+v e}$ and $\mathrm{PCR}^{-v e}$} \\
\hline $\mathrm{F}$ & Low & $0 / 841(0.0 \%)$ & 4/169 (2.4\%) & 6.14 (1.38-27.21); $P<0.01$ \\
\hline $\mathrm{F}$ & Medium & 4/669 (0.6\%) & 3/175 (1.7\%) & 2.69 (0.55-13.23); $P=0.420 .63$ \\
\hline $\mathrm{F}$ & High & 18/3343 (0.5\%) & 4/1294 (0.3\%) & $0.74(0.21-2.61) ; P=0.960 .09$ \\
\hline M & Low & 11/1339 (0.8\%) & 5/267 (1.9\%) & 2.45 (0.63-9.55); $P=0.360 .54$ \\
\hline M & Medium & 9/1010 (0.9\%) & 3/283 (1.1\%) & 1.07 (0.25-4.66); $P=1.000 .32$ \\
\hline $\mathrm{M}$ & High & $33 / 4450(0.7 \%)$ & 4/1739 (0.2\%) & $0.29(0.09-0.98) ; P=0.040 .01$ \\
\hline
\end{tabular}

$695 \quad{ }^{1}$ For those in the HCV antibody ${ }^{-v e}$ cohort, HCV diagnosis date was taken to be the

696 same as their respective HCV antibody ${ }^{+v e}$ cohort members, for the purpose of

697 analysis.

$698{ }^{2}$ Based on the likelihood-ratio test comparing the antibody ${ }^{+v e}$ cohort to the

699 antibody ${ }^{-v e}$ cohort, interaction-terms other than sex $\times$ HCV and deprivation $\times$

700 HCV were deemed not statistically significant and therefore excluded from the

701 final model.

$702 \quad{ }^{3}$ Adjusted OR and $P$ for exposure to HCV-infection within strata of sex and social

703 deprivation. Odds ratios adjusted for age at HCV diagnosis, year of HCV

704 diagnosis and the extra correlation due to the matching.

$705 \quad{ }^{4}$ Synergy Index. 
707 Table 4: Odds of having a diagnosis of T2DM in the period within \pm 1 year of the time 708 of HCV diagnosis in the HCV antibody ${ }^{+v e}$ cohort (total and broken down by PCR 709 status) compared to the HCV antibody ${ }^{-v e}$ cohort $^{1,2}$

710

\begin{tabular}{|c|c|c|c|}
\hline Sex & $\begin{array}{l}\text { Diabetes }^{+v e} \\
/ \mathrm{HCV} \mathrm{Ab}^{-v e}\end{array}$ & $\begin{array}{l}\text { Diabetes }^{+v e} \\
/ \mathrm{HCV} \mathrm{Ab}^{+v e}\end{array}$ & $\begin{array}{r}\mathrm{aOR}^{3} \\
(95 \% \mathrm{CI} ; P)\end{array}$ \\
\hline \multicolumn{4}{|c|}{ Antibody ${ }^{+v e}$} \\
\hline $\mathrm{F}$ & $36 / 20,626(0.2 \%)$ & 46/6996 (0.7\%) & 3.78 (2.29-6.25); $P<0.01$ \\
\hline $\mathrm{M}$ & $142 / 43,406(0$. & & $1.97(1.46-2.65) ; P<0.010 .71$ \\
\hline \multicolumn{4}{|c|}{ Antibody $^{+v e}$ and $\mathrm{PCR}^{+v e}$} \\
\hline $\mathrm{F}$ & $25 / 13,230(0.2$ & & 18); $P<0.01$ \\
\hline $\mathrm{M}$ & $111 / 30,223(0.4 \%)$ & 77/10,273 (0.7\%) & 2.07 (1.48-2.90); $P<0.010 .6$ \\
\hline \multicolumn{4}{|c|}{ Antibody $^{+v e}$ and PCR $^{-v e}$} \\
\hline $\mathrm{F}$ & 6/4591 (0.1\%) & $6 / 1555$ & $16.01) ; P=0.31$ \\
\hline $\mathrm{M}$ & $18 / 6283(0.3 \%)$ & $12 / 2131(0.6 \%)$ & $2.02(0.67-6.10) ; P=0.291 .01$ \\
\hline \multicolumn{4}{|c|}{$\begin{array}{l}{ }^{1} \text { For those in the HCV antibody }{ }^{-v e} \text { cohort, HCV diagnosis date was taken to be the } \\
\text { same as their respective HCV antibody }{ }^{+v e} \text { cohort members, for the purpose of } \\
\text { analysis. }\end{array}$} \\
\hline $\begin{array}{l}{ }^{2} \text { Based on the likelihood-ratio test comparing the antibody }{ }^{+v e} \text { cohort to the } \\
\text { antibody }{ }^{-v e} \text { cohort, interaction-terms other than sex } \times \text { HCV were deemed not } \\
\text { statistically significant and therefore excluded from the final model. }\end{array}$ & \multicolumn{3}{|c|}{$\begin{array}{l}{ }^{3} \text { Adjusted OR and } P \text { for exposure to HCV-infection within strata of sex. Odds ratios } \\
\text { adjusted for age at HCV diagnosis, year of HCV diagnosis, social deprivation and } \\
\text { the extra correlation due to the matching. } \\
\text { 4Svnergy Index. }\end{array}$} \\
\hline
\end{tabular}


714 Table 5: Hazard of being diagnosed with T2DM in the period $>1$ year after the time of 715 HCV diagnosis in the HCV antibody ${ }^{+v e}$ cohort (total and broken down by PCR status)

716 compared to the HCV antibody ${ }^{-v e}$ cohort $^{1,2}$

717

\begin{tabular}{|c|c|c|c|}
\hline Deprivatiol & $\begin{array}{l}\text { Diabetes }^{+v e} \\
/ \mathrm{HCV} \mathrm{Ab}^{-v e}\end{array}$ & $\begin{array}{l}\text { Diabetes }^{+v e} \\
/ \mathrm{HCV} \mathrm{Ab}^{+v e}\end{array}$ & $\begin{array}{r}\mathrm{aHR}^{3} \\
(95 \% \mathrm{CI} ; P)\end{array}$ \\
\hline \multicolumn{4}{|l|}{ Antibody $^{+v e}$} \\
\hline Low & $175 / 10,957$ & 61/2401 (2.5\%) & 1.53 (1.14-2.04); $P<0.01$ \\
\hline & & & $81-1.60) ; P=0.470 .7$ \\
\hline High & $646 / 34,470(1.9 \%)$ & 196/14,298 (1.4\%) & $0.88(0.75-1.03) ; P=0.110 .3$ \\
\hline \multicolumn{4}{|c|}{ Antibody $^{+v e}$ and $\mathrm{PCR}^{+v e}$} \\
\hline Low & $118 / 8158$ & $47 / 1750$ & 1.71 (1.21-2.40); $P<0.01$ \\
\hline & & & 86); $P=0.240 .7$ \\
\hline High & $470 / 25,027(1.9 \%)$ & $152 / 10,448(1.5 \%)$ & $0.89(0.74-1.07) ; P=0.220 .3$ \\
\hline \multicolumn{4}{|c|}{ Antibody $^{+v e}$ and PCR $^{-v e}$} \\
\hline Low & 25/1459 (1.7\%) & 9/376 (2.4\%) & 1.46 (0.68-3.13); $P=0.33$ \\
\hline $\mathrm{Mec}$ & 19/1395 & $4 / 401(1.0 \%)$ & $0.70(0.24-2.05) ; P=0.51 \quad(-)^{5}$ \\
\hline High & $91 / 6489(1.4 \%)$ & $36 / 2657$ (1.4\%) & $1.10(0.75-1.62) ; P=0.620 .53$ \\
\hline
\end{tabular}

${ }^{1}$ For those in the HCV antibody ${ }^{-v e}$ cohort, HCV diagnosis date was taken to be the same as their respective HCV antibody ${ }^{+v e}$ cohort members, for the purpose of analysis.

${ }^{2}$ Based on the likelihood-ratio test comparing the antibody ${ }^{+v e}$ cohort to the antibody ${ }^{-v e}$ cohort, interaction-terms other than deprivation $\times$ HCV were deemed not statistically significant and therefore excluded from the final model.

${ }^{3}$ Adjusted HR and $P$ for exposure to HCV-infection within strata of social deprivation. Odds ratios adjusted for age at HCV diagnosis, sex, year of HCV diagnosis and the extra correlation due to the matching.

${ }^{4}$ Synergy Index.

${ }^{5}$ To ease comparison between different models, the reference category (antibody ${ }^{-v e}$ and low deprivation) was fixed between models. This caused a negative (invalid) synergy index. 
720 Figure 1: Flowchart describing inclusion (boxes in the left column) and

721 exclusion criteria (boxes in the right column) for the HCV+ve cohort 


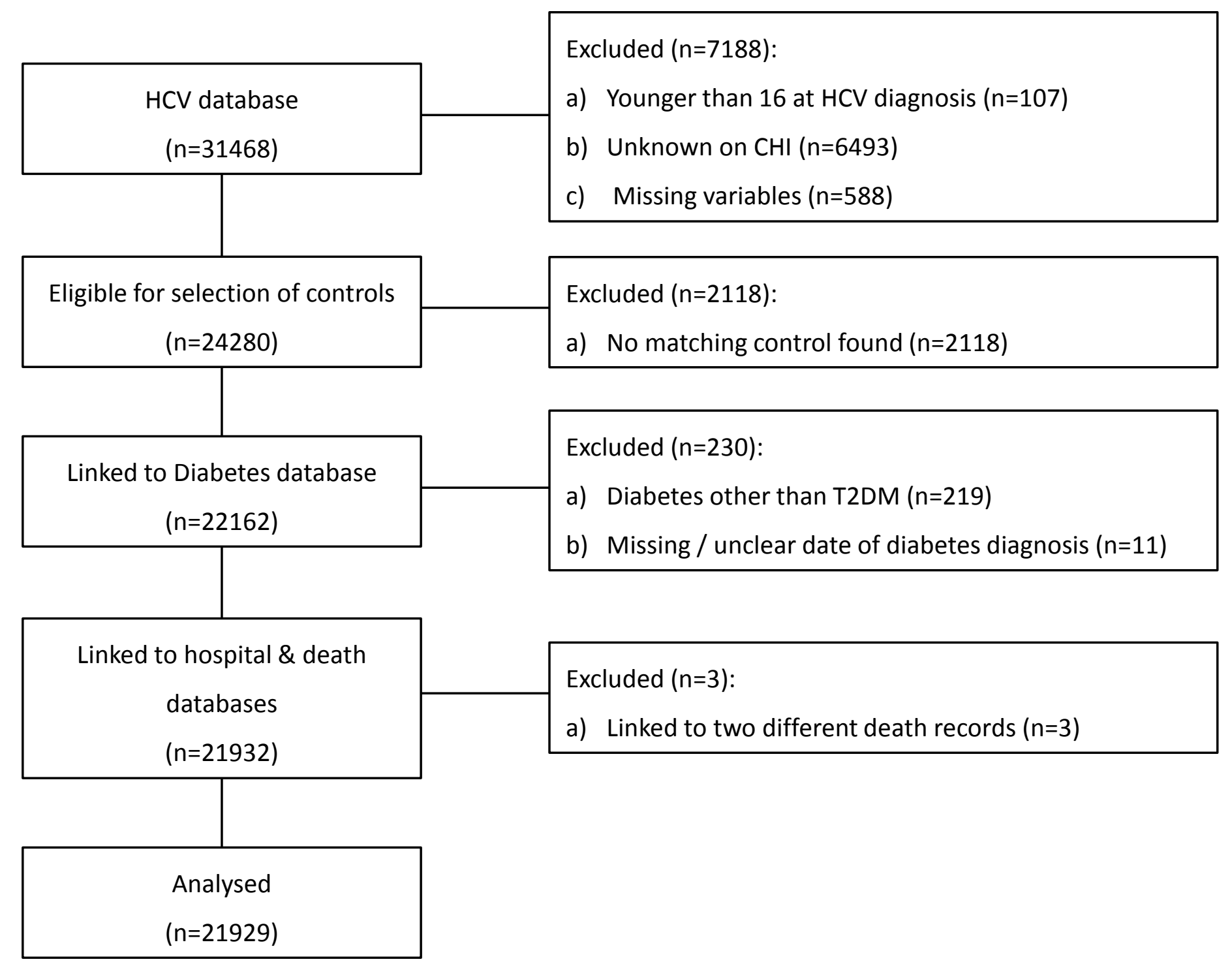

